Pacific Journal of Mathematic 


\title{
A DUALITY PRINCIPLE FOR RATIONAL APPROXIMATION
}

\author{
GeRHARD GIERZ AND BORIS SHEKHTMAN
}

\begin{abstract}
Let $E \subset C(K)$ be a subspace of continuous functions defined on a compact Hausdorff space $K$. We characterize those spaces for which the rational functions with denominators and numerators from $E$ are dense. Despite the non-linear structure of rational functions, this characterization uses only methods from linear functional analysis. As special cases, we recover various results on the density of Müntz rationals.
\end{abstract}

Introduction. Let $K$ be a compact Hausdorff space, and let $C(K)$ be the space of real-valued continuous functions on $K$. For an arbitrary subspace $E \subset C(K)$ let

$$
R(E)=\left\{\frac{g}{h}: g, h \in E ; h(k)>0 \text { for all } k \in K\right\}
$$

be the set of rational functions with respect to $E$. In this paper we investigate conditions on the space $E$ equivalent to the property that $R(E)$ is dense in $C(K)$. This problem is motivated by an increasing number of interesting results in approximation theory concerning rational approximations (cf. [4], [6]), and in particular by the question of D. J. Newman as to what makes the rationals dense: 'Apparently rational functions always want to be dense. There is something magical about performing that one division' ([4], p. 12). In this paper we propose a new approach to this problem:

Despite the very non-linear (not even convex) structure of $R(E)$ there is some linearity built into the set of rationals. The following observation opens some avenues for classical (linear) analysis to be used here:

$$
a=\frac{b}{c}=\frac{d}{e} \quad \text { implies } \quad a=\frac{b+d}{c+e} \quad \text { provided } c+e \neq 0 .
$$

In particular, the main result in the first section (Theorem 1) gives the following duality principle:

$R(E)$ is dense in $C(K)$ if and only if $R(F)$ is dense in $C(K)$ for every subspace $F, E \subset F$, of codimension no greater than 2 . 
This result resembles very much the classical duality principle (a linear subspace $E$ in any normed linear space is dense if and only if every hyperplane containing $E$ is dense).

The rest of $\$ 1$ contains numerous variations and corollaries of this principle. Section 2 consists of some examples. In the third section we use the duality principle to recover the results of G. Somorjai, J. Bak and D. J. Newman on Müntz rationals. In the last section we provide some additional remarks on the density of certain classes of rational functions.

In summary, this paper shows that the density of $R(E)$ can be completely decided from the structure of the linear bounded functionals on $C(K)$ which annihilate $E$.

1. Theory. We identify the set $\mathscr{M}(K)$ of all regular Borel measures on $K$ with the dual $C(K)^{*}$ of $C(K)$. If $\mu$ is a linear functional on $C(K)$ vanishing on $E$, we write $\mu \perp E$. For a measure $\mu$ on $K$ and a continuous function $\varphi \in C(K)$ we define a new measure $\varphi \mu$ on $K$ by the equation $\varphi \mu(f)=\mu(\varphi f)$ for all $f \in C(K)$.

1. THEOREM. The following conditions are equivalent for an arbitrary subspace $E \subset C(K)$.

(i) $R(E)$ is dense in $C(K)$.

(i') $R(E)$ is dense in $\{f \in C(K): 0 \leq f\}$.

(ii) For every (closed) linear subspace $F \subset C(K)$ such that $E \subset F$ and such that $\operatorname{codim} F \leq 2$, the set $R(F)$ is dense in $C(K)$.

(iii) For every pair of measures $\mu, \nu \perp E$ not both 0 , for every $\varphi \in C(K)$ with $0 \leq \varphi \leq 1$, and for every $\varepsilon>0$ we have $\varphi \mu+(1-\varphi) \nu \ngtr \varepsilon(|\mu|+|\nu|)$.

(iv) For every pair of measures $\mu, \nu \perp E$ not both 0 and for every $f \in C(K)$ we have $f \mu+\nu \ngtr|\mu|$.

(iv') For every pair of measures $\mu, \nu \perp E$ not both 0 and for every nonnegative $f \in C(K)$ we have $f \mu+\nu \ngtr|\mu|$.

(v) For every pair of measures $\mu, \nu \perp E$ not both 0 , for every $\varepsilon>0$, and for every $f \in C(K)$ there exists an $\tilde{f} \in C(K)$ such that $\|f-\tilde{f}\|<\varepsilon$ and such that $\tilde{f} \mu+\nu$ is neither positive nor negative.

$\left(v^{\prime}\right)$ For every pair of measures $\mu, \nu \perp E$ not both 0 , for every $\varepsilon>0$, and for every nonnegative $f \in C(K)$ there exists an $\tilde{f} \in C(K)$ such that $\|f-\tilde{f}\|<\varepsilon$ and such that $\tilde{f} \mu+\nu$ is neither positive nor negative.

Proof. (i') $\Rightarrow$ (i): Suppose that $f \in C(K)$ is given. Then $f+\|f\| 1$ is positive. Hence for every given $\varepsilon>0$ there are functions $g, h \in E$ such that $\|f+\| f\|1-g / h\|<\varepsilon$. It follows that $\|f-(g-\|f\| h) / h\|<\varepsilon$. 
(i) $\Rightarrow$ (ii) is trivial.

(ii) $\Rightarrow$ (iii): Assume that there exists measures $\mu, \nu \perp E$ not both 0 , $\varepsilon>0$ and $\varphi \in C(K), 0 \leq \varphi \leq 1$, such that $\varphi \mu+(1-\varphi) \nu \geq \varepsilon(|\mu|+|\nu|)$. Let $F=\operatorname{ker} \mu \cap \operatorname{ker} \nu$. Clearly, $E \subset F$ and $F$ has codimension 2 . We show that $R(F)$ cannot be dense. In fact, we will show that the $\varepsilon^{\prime}$-ball around $\varphi$ does not intersect $R(F)$, whenever $0<\varepsilon^{\prime}<\varepsilon$ :

Pick any function $g \in C(K)$ with $\|g\| \leq \varepsilon^{\prime}<\varepsilon$ we have $\varepsilon(|\mu|+|\nu|)$ $>\varepsilon^{\prime}(|\mu|+|\nu|) \geq g(\mu-\nu)$. It follows that $(\varphi-g)(\mu-\nu)+\nu>0$ whenever $\|g\| \leq \varepsilon^{\prime}$. Suppose that $(\varphi-g)=\alpha / \beta$, where $\|g\| \leq \varepsilon^{\prime}, \alpha, \beta \in$ $F$, and $\beta(k)>0$ for all $k \in K$. Then we obtain

$$
\begin{aligned}
0 & =(\mu-\nu)(\alpha)+\nu(\beta)=(\mu-\nu)((\varphi-g) \beta)+\nu(\beta) \\
& =[(\varphi-g)(\mu-\nu)+\nu](\beta)>0,
\end{aligned}
$$

a contradiction! (The last inequality follows from the fact that $\beta(k)$ is strictly positive for all $k \in K$ and the positivity of $(\varphi-g)(\mu-\nu)+\nu$.) Hence the $\varepsilon^{\prime}$-ball around $\varphi$ does not intersect $R(F)$.

(iii) $\Rightarrow$ (iv): Again, we assume that (iv') is not true. Then we can find a positive function $f \in C(K)$ such that $f \mu+\nu \geq|\mu|$. Hence, for all $g \in C(K)$ with $\|g\| \leq 1$ we have $f \mu+\nu \geq|\mu| \geq g \mu$. Especially, if $\|g\| \leq$ $1 / 3$, then $\|g-2 / 3\| \leq 1$, hence $(f+2 / 3-g) \mu+\nu \geq 0$. Moreover, since $\mu / 3=\sup \{g \mu:\|g\| \leq 1 / 3\}$, we have $(f+2 / 3) \mu+\nu \geq \mu / 3$. Let $f_{0}=f+2 / 3$. We obtain

$$
f_{0} \mu+\nu \geq \frac{1}{3} \mu ; \quad f_{0}(k) \geq \frac{2}{3} \quad \text { for all } k \in K .
$$

Moreover, for every $\tilde{f}$ in the ball with radius $1 / 3$ we have $\tilde{f}(k) \geq 1 / 3>0$ and $\tilde{f} \mu+\nu>0$. Let $g_{0}=1 / f_{0}$ and choose $M$ such that $\left\|f_{0}\right\| \leq M / 3$. We claim that

$$
\mu+g_{0} \nu \geq \frac{1}{(M+1)\left\|f_{0}\right\|}|\nu| ; \quad g_{0}(k) \geq \frac{1}{\left\|f_{0}\right\|} \quad \text { for all } k \in K .
$$

Clearly, $g_{0}(k)=1 / f_{0}(k) \geq 1 /\left\|f_{0}\right\|$ for every $k \in K$. Now assume that $\|\tilde{g}-g\| \leq 1 /(M+1)\left\|f_{0}\right\|$. Then $\tilde{g}(k) \geq M /(M+1)\left\|f_{0}\right\|>0$ and

$$
\begin{aligned}
\left|f_{0}(k)-\frac{1}{\tilde{g}(k)}\right| & =\frac{f_{0}(k)}{\tilde{g}(k)}\left|\tilde{g}(k)-\frac{1}{f_{0}(k)}\right|=\frac{f_{0}(k)}{\tilde{g}(k)}\left|\tilde{g}(k)-g_{0}(k)\right| \\
& \leq \frac{(M+1)\left\|f_{0}\right\|^{2}}{M} \frac{1}{(M+1)\left\|f_{0}\right\|} \leq \frac{\left\|f_{0}\right\|}{M} \leq \frac{1}{3}
\end{aligned}
$$


for all $k \in K$. It follows that $\left\|f_{0}-1 / \tilde{g}\right\| \leq 1 / 3$, and thus $(1 / \tilde{g}) \mu+\nu \geq 0$. This clearly implies $\mu+\tilde{g} \nu \geq 0$ whenever $\tilde{g}$ belongs to the ball of radius $1 /(M+1)\left\|f_{0}\right\|$ with center $g_{0}$. Using again the equation $|\nu|=$ $\sup \{g \nu:\|g\| \leq 1\}$, this yields

$$
\mu+g_{0} \nu \geq \frac{1}{(M+1)\left\|f_{0}\right\|}|\nu|
$$

If we let

$$
\rho=\min \left\{\frac{1}{3}, \frac{1}{(M+1)\left\|f_{0}\right\|}\right\}
$$

then we obtain $f_{0} \mu+\nu \geq \rho|\mu|$ and $\mu+g_{0} \nu \geq \rho|\nu|$, thus

$$
\left(1+f_{0}\right) \mu+\left(1+g_{0}\right) \nu \geq \varphi(|\mu|+|\nu|) .
$$

Now let

$$
\varphi=\frac{\left(1+f_{0}\right)}{\left(2+f_{0}+g_{0}\right)}
$$

and

$$
\varepsilon=\frac{\rho}{2+\left\|f_{0}\right\|+\left\|g_{0}\right\|}
$$

It follows that $\varphi \mu+(1-\varphi) \nu \geq \varepsilon(|\mu|+|\nu|)$, contradicting (iii).

$\left(\mathrm{iv}^{\prime}\right) \Rightarrow\left(\mathrm{v}^{\prime}\right)$ : Assume that there is a function nonnegative $f \in C(K)$, a pair of measures $\mu, \nu \perp E$ and an $\varepsilon>0$ such that for every $\tilde{f}$ with $\|\tilde{f}-f\|<\varepsilon$ the linear functional $\tilde{f} \mu+\mu$ is either positive or negative. Consider the set

$$
A=\{\tilde{f} \mu+\nu:\|\tilde{f}-f\| \leq \varepsilon\}=f \mu+\nu+\{g \mu:\|g\| \leq \varepsilon\} .
$$

Then $A$ is convex. Hence, if $A$ contains strictly positive and strictly negative elements, $A$ has to be a line segment containing 0 . (Indeed, assume that $\gamma_{1} \in A$ is strictly positive and that $\gamma_{2} \in A$ is strictly negative. If $\gamma_{1}$ and $\gamma_{2}$ were not linearly dependent, then for every $0 \leq r \leq 1$ we have either $r \gamma_{1}+(1-r) \gamma_{2}>0$ or $r \gamma_{1}+(1-r) \gamma_{2}<0$. Hence $\left\{r: r \gamma_{1}\right.$ $\left.+(1-r) \gamma_{2} \geq 0\right\}$ and $\left\{r: r \gamma_{1}+(1-r) \gamma_{2} \leq 0\right\}$ are non-empty disjoint closed sets covering the unit interval, a contradiction. It follows that every such pair of elements of $A$ is linearly dependent, and therefore $A$ has to be a line segment containing 0 .) Since $0 \in A$, we can find a function $h$ such that $f \mu+\nu=h \mu$, i.e., $A=h \mu+\{g \mu:\|g\| \leq \varepsilon\}$. Moreover, since $0 \in A$, we may assume w.l.o.g. that $\|h\| \leq \varepsilon$. Consider the elements 
$(h+\varepsilon) \mu,(h-\varepsilon) \mu \in A$. If both were positive, we would obtain $h \mu \geq-\varepsilon \mu$ and $h \mu \geq+\varepsilon \mu$, i.e. $h \mu \geq \varepsilon|\mu| \geq g \mu$ for every $g \in C(K)$ with $\|g\| \leq \varepsilon$. This would imply $\gamma \geq 0$ for every $\gamma \in A$, contradicting our assumptions. Similarly, if both $(h+\varepsilon) \mu$ and $(h-\varepsilon) \mu$ were negative, we would arrive at the contradiction $\gamma \leq 0$ for every $\gamma \in A$. Hence we have either $(h+\varepsilon) \mu$ $\leq 0$ and $(h-\varepsilon) \mu \geq 0$ or vice versa. In the first case, we obtain $h \mu \leq-\varepsilon \mu$ and $-h \mu \leq-\varepsilon \mu$, i.e. $|h \mu| \leq-\varepsilon \mu$. This implies that $-\mu$ is positive. The second case yields $\mu \geq 0$. In any case, we would find a positive measure $\mu^{\prime} \perp E$, contradicting (vi') with $f=1$ and $\nu=0$.

Hence, $A$ contains either only positive or only negative elements, w.l.o.g. $\tilde{f} \mu+\nu \geq 0$ whenever $\|\tilde{f}-f\|<\varepsilon$. This implies $f \mu+\nu \geq g \mu$ for all $g \in C(K),\|g\|<\varepsilon$. Since $\sup \{g \mu:\|g\|<\varepsilon\}=\varepsilon|\mu|$, we conclude that $f \mu+\nu \geq \varepsilon|\mu|$, i.e. $(f / \varepsilon) \mu+(\nu / \varepsilon) \geq|\mu|$, contradicting (iv').

$\left(\mathrm{v}^{\prime}\right) \Rightarrow\left(\mathrm{iv}^{\prime}\right)$ : Suppose we could find a pair of measures $\mu, \nu \perp E$ and a function $f \in C(K)$ such that $f \mu+\nu \geq|\mu|$. Then for every $g \in C(K)$ with $\|g\|<1$ we would have $f \mu+\nu \geq|\mu| \geq g \mu$, i.e. $(f-g) \mu+\nu \geq 0$, contradicting $\left(\mathrm{v}^{\prime}\right)$ with $\varepsilon=1$.

$\left(\mathrm{v}^{\prime}\right) \Rightarrow\left(\mathrm{i}^{\prime}\right)$ : Suppose that $R(E)$ is not dense in $\{f \in C(K): 0 \leq f\}$. Then we can find a nonnegative function $f \in C(K)$ and an $\varepsilon>0$ such that the $\varepsilon$-ball around $f$ does not intersect $R(E)$. Consider the following subset of $C(K) \times C(K)$ :

$$
C_{f, \varepsilon}=\{(g, h): h(k)>0 \text { for all } k \in K ;\|f-g / h\|<\varepsilon\} .
$$

The equivalence $\|f-g / h\|<\varepsilon$ if and only if $-\varepsilon h(k)<f(k) h(k)-g(k)$ $<\varepsilon h(k)$ for all $k \in K$ shows that $C_{f, \varepsilon}$ is an open convex subset of $C(K) \times C(K)$. By assumption, $C \cap E \times E=\varnothing$. Hence we can find measures $\mu$ and $\nu$ such that

(a) $E \times E \subset\{(\alpha, \beta): \mu(\alpha)+\nu(\beta)=0\}$;

(b) $C_{f, \varepsilon} \cap\{(\alpha, \beta): \mu(\alpha)+\nu(\beta)=0\}=\varnothing$.

Clearly, (a) implies that $E \subset \operatorname{ker} \mu \cap \operatorname{ker} \nu$, i.e. $\mu, \nu \perp E$ and clearly $\mu$ and $\nu$ cannot be both equal to 0 . We will show next that $\tilde{f} \mu+\nu$ is either positive or negative whenever $\|f-\tilde{f}\|<\varepsilon$ : Assume that for one such $\tilde{f}$, the linear functional $\tilde{f} \mu+\nu$ is neither positive nor negative. Then we can find a strictly positive function $h$ such that $[\tilde{f} \mu+\nu](h)=0$. Let $g=\tilde{f} h$. We find $(g, h) \in C_{f, \varepsilon} \cap\{(\alpha, \beta): \mu(\alpha)+\nu(\beta)=0\}$, contradicting (b).

(ii) $\Rightarrow$ (iv) is similar to (ii) $\Rightarrow$ (iii).

(iv) $\Leftrightarrow$ (v) is completely analogous to $\left(\mathrm{iv}^{\prime}\right) \Leftrightarrow\left(\mathrm{v}^{\prime}\right)$.

(v) $\Rightarrow\left(v^{\prime}\right)$ is trivial. 
Let us consider some special cases.

If $\mu$ is a measure on a compact space $K$, and if $f \in C(K), f \neq 0$, is given such that $\mu(f)=\|\mu\|\|f\|$, then we say that $\mu$ peaks at $f$. It is easy to see that $f \mu \geq|\mu|$ implies that $\mu$ peaks at $(-1) \vee(f \wedge 1)$. Moreover, $\mu$ peaks at $f$ for some $f \in C(K)$ if and only if $\operatorname{supp} \mu_{+} \cap \operatorname{supp} \mu_{-}=\varnothing$.

It was shown in [3] that for a hyperplane $E=\operatorname{ker} \mu$ the set $R(E)$ is dense if and only if $\mu$ does not peak. For subspace of codimension 2 we have

2. Corollary. Let $\mu, \nu$ be two measures on $K$ neither of which is peaking, and assume that there is a point $x \in\left(\operatorname{supp} \mu_{+} \cap \operatorname{supp} \mu_{-}\right) \backslash \operatorname{supp} \nu$. If $E=\operatorname{ker} \mu \cap \operatorname{ker} \nu$, then $R(E)$ is dense.

Proof. Assume not. Then, by Theorem 1, we can find an $\varepsilon>0$, a function $f \in C(K)$ and constants $r, s, a, b \in \Re$ such that

$$
f(r \mu+s \nu)+(a \mu+b \nu) \geq \varepsilon|r \mu+s \nu| .
$$

Let $A$ be a closed neighborhood of $x$ disjoint from $\operatorname{supp} \nu$. Since restriction of measures to closed sets is a lattice homomorphism, we find

$$
r(f \mid A)(\mu \mid A)+a(\mu \mid A) \geq \varepsilon|r(\mu \mid A)| .
$$

If $r=0$, then $a \mu \mid A$ would be positive, which is only possible if $a=0$, since $x \in A \cap \operatorname{supp} \mu_{+} \cap \operatorname{supp} \mu_{-}$. It would follow that $f s \nu+b \nu \geq \varepsilon|s \nu|$. Hence either $b \nu$ would be positive (if $s=0$ ) of $\left(f_{s}+b\right)(\varepsilon|s|)^{-1} \nu \geq|\nu|$, in which case $\nu$ would peak by the above remark. We conclude that $r \neq 0$. Hence

$$
\frac{r(f \mid A)+a}{\varepsilon|r|}(\mu \mid A) \geq|(\mu \mid A)|,
$$

i.e. $\mu \mid A$ would peak, contradicting the fact that $x \in \operatorname{supp}(\mu \mid A)_{+} \cap$ $\operatorname{supp}(\mu \mid A)$.

3. Corollary. Let $E \subset C(K)$ be a linear subspace and assume that there is a point $x_{0} \in K$ such that $x_{0} \in \operatorname{supp} \mu_{+} \cap \operatorname{supp} \mu_{-}$for every $\mu \perp E$. Then $R(E)$ is dense.

Proof. Assume not. Then by condition (v) of Theorem 1 there exist measures $\mu, \nu \perp E$, there exists $\varepsilon>0$ and there exists a function $f \in C(K)$ such that $\tilde{f} \mu+\nu$ is either positive or negative for every $\tilde{f} \in B_{\varepsilon}(f)$. Pick a function $\tilde{f}$ such that $\|\tilde{f}-f\|<\varepsilon$ and such that $\tilde{f}$ is constant on a closed 
neighborhood $A$ of $x_{0}$. Let $r=\tilde{f}\left(x_{0}\right)$. Then

$$
(r \mu+\nu)|A=(\tilde{f} \mu+\nu)| A \geq \varepsilon|\mu| A \mid \geq 0,
$$

contradicting the fact that $x_{0} \in \operatorname{supp}(r \mu+\nu)_{+} \cap \operatorname{supp}(r \mu+\nu)_{-}$.

4. Corollary. Let $K=\{1 / n: n \in \mathbf{N}\} \cup\{0\}$. Then for a linear subspace $E \in C(K), R(E)$ is dense if and only if no measure $\mu \perp E$ peaks.

Proof. Since 0 is the only point of $K$ which is not isolated, a measure $\mu$ on $K$ does not peak if and only if $0 \in \operatorname{supp} \mu_{+} \cap \operatorname{supp} \mu_{-}$. Hence the result follows from Corollary 3.

We will see that Corollary 4 is not true in general. As a matter of fact, it is never true in a space $K$ which has two accumulation points.

2. Examples. Our first example shows that it may happen that $R(F)$ is dense in $C(K)$ for every hyperplane $F$ containing $E$, but $R(E)$ itself is not dense:

1. ExAmple. Let $K$ be the real interval between 0 and 5. We define two measures $\mu$ and $\nu$ by

$$
\mu(g)=\int_{0}^{1} g(x) d x-\int_{1}^{2} g(x) d x+\int_{3}^{4} g(x) d x-\int_{4}^{5} g(x) d x
$$

and

$$
\nu(g)=\int_{0}^{2} g(x) d x-2 \int_{3}^{4} g(x) d x+4 \int_{4}^{5} g(x) d x .
$$

Let $E=\operatorname{ker} \mu \cap \operatorname{ker} \nu$.

Firstly, we check that $R(E)$ is not dense: Pick a continuous function $f \in C[0,5]$ such that $f(x)=0$ for $0 \leq x \leq 2$ and $f(x)=3$ for $3 \leq x \leq 5$. Then

$$
\begin{aligned}
(f \mu+\nu)(g) & =\mu(f g)+\nu(g) \\
& =\int_{0}^{2} g(x) d x+\int_{3}^{5} g(x) d x=|\mu|(g),
\end{aligned}
$$

i.e., $f \mu+\nu=|\mu|$. Hence $R(E)$ is not dense by (1.1). Now consider any hyperplane $F$ containing $E$. Then $F=\operatorname{ker} \gamma$, where $\gamma=r \mu+s \nu$ for suitable $r, s \in \Re$. It turns out that for every choice of $r$ and $s$, either $1 \in \operatorname{supp} \gamma_{+} \cap \gamma_{-}$or $4 \in \operatorname{supp} \gamma_{+} \cap \operatorname{supp} \gamma_{-}$. Hence $R(F)$ is dense by [3]. 
2. ExAmple. Let $K=[0,2 \pi]$ be the set of real numbers between 0 and $2 \pi$, and let $E \subset C(K)$ be given as follows:

$$
E=\left\{f \in C(K): \int_{0}^{2 \pi} f(x) \sin x d x=\int_{0}^{2 \pi} f(x) \cos x d x=0\right\} .
$$

We will show that $R(E)$ is dense in $C([0,2 \pi])$. First of all, note that every measure $\mu$ orthogonal to $E$ is of the form

$$
\begin{aligned}
\mu(g) & =\int_{0}^{2 \pi} g(x)(a \cos x+b \sin x) d x \\
& =r \int_{0}^{2 \pi} g(x) \cos (x+\alpha) d x
\end{aligned}
$$

for certain constants $r \in \Re$ and $\alpha \in[0,2 \pi]$. Hence it follows from the result in [3] that for every hyperplane $F$ containing $E$, the set $R(F)$ is dense in $C([0,2 \pi])$. Hence, if $R(E)$ were not dense, then we could find linearly independent measures $\mu, \nu \perp E$, a continuous function $\varphi \in$ $C([0,2 \pi]), 0 \leq \varphi \leq 1$, and $\varepsilon>0$ such that

$$
\varphi \mu+(1 \varphi) \nu \geq \varepsilon(|\mu|+|\nu|) \text {. }
$$

Representing $\mu$ as $\mu(g)=r_{0} \int_{0}^{2 \pi} g(x) \cos \left(x+a_{0}\right) d x$ and $\nu(g)=$ $r_{1} \int_{0}^{2 \pi} g(x) \cos \left(x+\alpha_{1}\right) d x$, we obtain

$$
\begin{aligned}
& \varphi(x) r_{0} \cos \left(x+\alpha_{0}\right)+(1-\varphi(x)) r_{1} \cos \left(x+\alpha_{1}\right) \\
& \geq \varepsilon\left(\left|r_{0} \cos \left(x+\alpha_{0}\right)\right|+\left|r_{1} \cos \left(x+\alpha_{1}\right)\right|\right) .
\end{aligned}
$$

It follows that $r_{0} \cos \left(x+\alpha_{0}\right)$, and $r_{1} \cos \left(x+\alpha_{1}\right)$ cannot be simultaneously negative, and hence $\alpha_{0}-\alpha_{1}$ is a multiple of $\pi$, contradicting the fact that $\mu$ and $\nu$ are linearly independent.

3. EXAMPLE. Let $K$ be the set of real numbers between 0 and $2 \pi$ and define $E \subset C(K)$ by

$$
E=\left\{f \in C(K): \int_{0}^{2 \pi} f(x) \sin x d x=\int_{0}^{2 \pi} f(x) \cos 2 x d x=0\right\} .
$$

Then, contrary to Example 2, $R(E)$ is not dense in $C(K)$. If we define $\mu(f)=\int_{0}^{2 \pi} f(x) \sin x d x$ and $\nu(f)=\int_{0}^{2 \pi} f(x) \cos 2 x d x$, then $\mu$ and $\nu$ are orthogonal to $E$, but for $f(x)=2 \sin x$ we have

$$
\begin{aligned}
f \mu+\nu & =2 \sin ^{2} x+\cos 2 x \\
& =(1-\cos 2 x)+\cos 2 x=1 \\
& \geq|\sin x|=|\mu|,
\end{aligned}
$$

hence $R(E)$ is not dense by Theorem 1.(iv). 
3. Approximation by Müntz Rationals. In this section we will use our approach to recover the results of G. Somorjai [5] and J. Bak and D. J. Newman [1] on approximation with Müntz rationals. We will start with an abstract version of the theorem of Somorjai:

1. Definition. Let $0 \leq a \leq 1$ and $\varepsilon>0$. A function $z_{\varepsilon, a} \in C[0,1]$ is called an $\varepsilon$-zoomer at $a$ if $z_{\varepsilon, a}(r) \geq 0$ for all $0 \leq r \leq 1$ and

(i) $z_{\varepsilon, a}(x) \geq 1 / \varepsilon$ for $x \geq a+\varepsilon$;

(ii) $z_{\varepsilon, a}(x) \leq \varepsilon$ for $x \leq a-\varepsilon$.

2. ThEOREM (cf. [5]). Let $E \subset C[0,1]$ be a subspace such that $E$ contains an $\varepsilon$-zoomer at a for all $a \in[0,1]$ and for all $\varepsilon>0$. Then $R(E)$ is dense in $C[0,1]$.

Proof. Let $\mu \perp E, \mu \neq 0$. We first show that

$$
t_{0}=\sup \{t: t \in \operatorname{supp} \mu\} \in \operatorname{supp} \mu_{+} \cap \operatorname{supp} \mu_{-} .
$$

For a proof of this statement, it is evidently sufficient to show that for any neighborhood $U$ containing the point $t_{0}$, the restriction $\mu \mid U$ is neither negative nor positive. Contrary to it, let us assume that there exists an interval $\left[t-3 \eta, t_{0}\right], \eta>0$, where the measure $\mu$ is strictly positive. Then it is also strictly positive on the interval $\left[t_{0}-\eta, t_{0}\right]$, hence $\mu\left(\left[t_{0}-\eta, t_{0}\right]\right)$ $\geq \delta$ for some $\delta>0$. For every $\varepsilon \in(0, \eta]$ we have

$$
\begin{aligned}
0= & \mu\left(z_{\varepsilon, t_{0}-\eta}\right) \\
= & \int_{0}^{t_{0}-3 \eta} z_{\varepsilon, t_{0}-\eta}(s) d \mu(s)+\int_{t_{0}-3 \eta}^{t_{0}-\eta} z_{\varepsilon, t_{0}-\eta}(s) d \mu(s) \\
& +\int_{t_{0}-\eta}^{1} z_{\varepsilon, t_{0}-\eta}(s) d \mu(s) \\
= & \int_{0}^{t_{0}-3 \eta} z_{\varepsilon, t_{0}-\eta}(s) d \mu(s)+\int_{t_{0}-3 \eta}^{t_{0}-\eta} z_{\varepsilon, t_{0}-\eta}(s) d \mu(s) \\
& +\int_{t_{0}-\eta}^{t_{0}} z_{\varepsilon, t_{0}-\eta}(s) d \mu(s) \\
\geq & \int_{0}^{t_{0}-3 \eta} z_{\varepsilon, t_{0}-\eta}(s) d \mu(s)+\int_{t_{0}-\eta}^{t_{0}} z_{\varepsilon, t_{0}-\eta}(s) d \mu(s) .
\end{aligned}
$$

Letting $\varepsilon$ tend to 0 we have (by definition)

$$
\int_{0}^{t_{0}-3 \eta} z_{\varepsilon, t_{0}-\eta}(s) d \mu(s) \rightarrow 0
$$


while

$$
\int_{t_{0}-\eta}^{t_{0}} z_{\varepsilon, t_{0}-\eta}(s) d \mu(s) \geq \frac{\delta}{\varepsilon} \rightarrow \infty,
$$

which gives us a contradiction.

To finish the proof of the Theorem, we have to show that for any pair of measures $\mu, \nu \perp E$,

$$
\overline{R(\operatorname{ker} \mu \cap \operatorname{ker} \nu)}=C[0,1] .
$$

We consider two cases:

(1) Suppose that for some pair of measures

$$
\nu_{1}=a_{1} \mu+\beta_{1} \nu ; \quad \nu_{2}=\alpha_{2} \mu+\beta_{2} \nu
$$

we have $\sup \left(\operatorname{supp} \nu_{1}\right)>\sup \left(\operatorname{supp} \nu_{2}\right)$. Then we have $t_{0}=\sup \left(\operatorname{supp} \nu_{1}\right) \in$ $\operatorname{supp} \nu_{1} \cap \operatorname{supp} \nu_{1-}$ and $t_{0} \notin \operatorname{supp} \nu_{2}$. Thus (2) holds by Corollary (1.2).

(2) Suppose that $t_{0}=\sup (\operatorname{supp} \nu)=\sup (\operatorname{supp} \alpha \mu+\beta \nu)$ for all $\alpha, \beta$ $\in \Re$. Then $t_{0} \in \operatorname{supp}(\alpha \mu+\beta \nu)_{+} \cap \operatorname{supp}(\alpha \mu+\beta \nu)_{-}$for all $\alpha, \beta$, hence (2) follows from Corollary (1.3).

We are now in the position to prove the following

3. TheOREM. (Somorjai [5], Bak and Newman [1].) Let $\Lambda$ be an infinite set of positive real numbers containing 1 . Let

$$
E=\operatorname{span}\left\{t^{\lambda}: \lambda \in \Lambda\right\} \subset C[0,1] .
$$

Then $R(E)$ is dense in $C[0,1]$.

Proof. We consider three cases:

(I) $\Lambda$ contains a strictly decreasing sequence $\left(\lambda_{j}\right)$ such that $\lim _{j \rightarrow \infty} \lambda_{j}$ $=0$. We want to show that $0 \in \operatorname{supp} \mu_{+} \cap \operatorname{supp} \mu_{-}$for every $0 \neq \mu \perp E$. (In this case, the result follows from Corollary 3.)

Firstly, we show that for every $\varepsilon>0$

$$
\mu \mid[0, \varepsilon] \neq 0 .
$$

Suppose not. Consider the function

$$
f(z)=\int_{0}^{1} t^{z} d \mu(t)=\int_{\varepsilon}^{1} t^{z} d \mu(t), \quad z \in \mathbf{C} .
$$

Then $f(z)$ is an entire function (cf. [2]), and $f\left(\lambda_{j}\right)=0$ for all $j$. Hence the zeros of $f$ have an accumulation point. Consequently, $f(z)=0$ for all complex numbers $z$ and therefore $\mu=0$. 
It now remains to show that for every $\eta>0$ such that $3 \eta<e^{-1}$ the restriction of $\mu$ to the interval $[0,3 \eta]$ is neither positive nor negative. Suppose that

$$
(\mu \mid[0,3 \eta])>0 .
$$

We first observe that $\mu(0)=0$. Indeed,

$$
0=\lim _{j \rightarrow \infty} \mu\left(1-t^{\lambda_{\jmath}}\right)=\mu(0)
$$

since $1-t^{\lambda} \rightarrow 0$ for $t \in(0,1]$. Hence the assumption (3) implies that $(\mu \mid[\delta, \eta])>0$ for every $0<\delta<\eta$.

Let $\varepsilon>0$ be given. Choose $k$ so large that $1-t^{\lambda_{k}}<\varepsilon$ for all $t \in[\delta, 1]$. Let $k=-\ln (2 \eta)$. Consider the function $P_{N}(t)=$ $N !\left[\lambda_{k}, \ldots, \lambda_{k+N}\right] t^{s}$ where $\left[\lambda_{k}, \ldots, \lambda_{k+N}\right] t^{s}$ is the $N$ th divided difference at $t^{s}$, where $t^{s}$ is viewed as a function of $s$ (cf. [2]). By the mean value theorem we can find a number $\lambda(t) \in\left[\lambda_{k+N}, \lambda_{k}\right]$ so that $P_{N}(t)=$ $t^{\lambda(t)}(\ln t)^{N}$. By the definition of divided differences, $P_{N}(t) \in E$ and $Q_{N}(t)=P_{N}(t) / K^{N}=t^{\lambda(t)}((\ln t) / K)^{N}$ has the following properties (for even $n$ ):

(i) $Q_{n}(t) \geq 0$;

(ii) $\lim _{n \rightarrow \infty} Q_{n}(t)=\infty$ for $t \in[\delta, \eta]$;

(iii) $\lim _{n \rightarrow \infty} Q_{n}(t)=0$ for $t \in[3 \eta, 1]$.

Similarly to the proof of (3.2) we have

$$
\begin{aligned}
0 & =\mu\left(Q_{n}\right)=\int_{0}^{3 \eta} Q_{n}(s) d \mu(s)+\int_{3 \eta}^{1} Q_{n}(s) d \mu(s) \\
& \geq \int_{s}^{\eta} Q_{n}(s) d \mu(s)-\left|\int_{3 \eta}^{1} Q_{n}(s) d \mu(s)\right| .
\end{aligned}
$$

Since the first integral tends to infinity and the second integral tends to zero, we have a contradiction.

(II) Assume that $\Lambda$ contains a sequence $\left(\lambda_{j}\right)$ such that $\lim _{j \rightarrow \infty} \lambda_{j}=$ $\infty$. In this case it is sufficient to observe that the functions $z_{\varepsilon, a}(t)=$ $(t / a)^{\lambda}, \in E$ are zoomers for sufficiently large $\lambda_{j}$. Hence this case is a consequence of (3.1).

(III) Finally if $\Lambda$ contains a sequence $\left(\lambda_{J}\right)$ which is bounded and bounded from below by $r>0$, then the Müntz-theorem (cf. [2]) implies that $E$ is dense in $C[0,1]$. Since $1 \in E$, we have $E \subset R(E)$ and consequently $\overline{R(E)}=C[0,1]$.

REMARK. It is interesting to note that in the proof of (3.3) we have used the same functions which were used in the constructive proof of 
theorem (cf. [2]). However they have been used for a somewhat different purpose.

Similar results can be obtained in higher dimensions. More specifically, let $\Lambda=\left(\lambda_{j}\right)_{j}$ be an increasing sequence of positive integers. Let $D$ be a compact subset of the positive cone in $\Re^{n}$. Let

$$
E=\operatorname{span}\left\{t_{i}^{\lambda_{i_{1}}} \cdot t_{2} i^{\lambda_{i_{2}}} \cdots \cdot t_{n} i^{\lambda_{i_{n}}}: \lambda_{i_{1}}, \ldots, \lambda_{i_{n}} \in \Lambda\right\} .
$$

Then $R(E)$ is dense in $C(D)$. As a matter of fact, if $\lim _{j \rightarrow \infty} \lambda_{j}=\infty$, and if $\mu \perp E$, then points in supp $\mu$ which have maximal distance to the origin belong to $\operatorname{supp} \mu_{+} \cap \operatorname{supp} \mu_{-}$. If $\lim _{j \rightarrow \infty} \lambda_{j}=0$ and if $D$ is in the interior of the positive cone, then $E$ is dense in $C(D)$. If $D$ is not in the interior of the positive cone, then every point in the support of $\mu$ with at least one coordinate equal to zero belongs to $\operatorname{supp} \mu_{+} \cap \operatorname{supp} \mu_{-}$.

These observations raise the interesting question of the density of $R(E \otimes E)$ in $C(K \times K)$. We intend to explore this problem in a subsequent paper.

4. Further remarks. Let us start this section by pointing out that Theorem (1.1) maybe also used 'pointwise': The proof of $\left(\mathrm{v}^{\prime}\right) \Rightarrow\left(\mathrm{i}^{\prime}\right)$ show that a function $f$ belongs to the closure of $R(E)$ provided that for every pair of measure $\mu, \nu \perp E$ and every $\varepsilon>0$ there exists a $\tilde{f}$ with $\|f-\tilde{f}\|<\varepsilon$ such that $\tilde{f} \mu+\nu$ is neither positive nor negative. This observation leads to a generalization of Corollary (1.3) that has a 'set of antisymmetry flavor':

1. Proposition. Let $E$ be a linear subspace of $C(K)$ and let $A$ be a subset of $K$ such that for every $\mu \perp E$ we have $A \cap \operatorname{supp} \mu_{+} \cap \operatorname{supp} \mu_{-} \neq \varnothing$. If $f \in C(K)$ and $f \mid A$ is a constant, then $f \in \overline{R(E)}$.

Proof. Assume that $f(k)=r_{0}$ for all $k \in A$. For a given $\varepsilon>0$ pick a function $\tilde{f}$ in the $\varepsilon$-neighborhood of $f$ such that $\tilde{f}(k)=r_{0}$ for a certain open neighborhood $U$ of $A$. We claim that $\tilde{f} \mu+\nu$ is neither positive nor negative whenever $\mu, \nu \perp E$. Assume not. By multiplying with -1 if necessary, we may assume that $\tilde{f} \mu+\nu>0$. This would imply that

$$
\left(r_{0} \mu+\nu\right)|U=(\tilde{f} \mu+\nu)| U \geq 0,
$$

contradicting the fact that

$$
A \cap \operatorname{supp}\left(r_{0} \mu+\nu\right)_{+} \cap \operatorname{supp}\left(r_{0} \mu+\nu\right)_{-} \neq \varnothing .
$$


The next result follows from (4.1):

2. Proposition. Let $\lambda$ be a Borel measure on $K$ and let $E$ be a linear subspace of $C(K)$ such that there exists a set $A$ of $\lambda$-measure 0 satisfying $A \cap \operatorname{supp} \mu_{+} \cap \operatorname{supp} \mu_{-} \neq \varnothing$ for each $\mu \perp E$. Then $R(E)$ is dense in $L_{p}(\lambda)$ for every $1 \leq p<\infty$.

Example (2.1) shows that $R(E)$ may be dense in $L_{p}(\lambda)$ for every $1 \leq p<\infty$ without being dense in $C(K)$.

Our last remark concerns Chebyshev Systems. Recall that an $n+1$ dimensional subspace $E \subset C([0,1])$ is called Chebyshev, provided that every non-zero function $f \in E$ has no more than $n$ zeros. D. J. Newman (see [4]) conjectured that for a space $E$ containing Chebyshev systems of arbitrary high dimensions, the set $R(E)$ is dense in $C([0,1])$. Our Theorem (1.1) shows that we only have to verify Newman's conjecture for spaces $E$ of codimension 1 and 2 . For spaces of codimension 1, the question can be solved:

3. Proposition. Let $E \subset C([0,1])$ be a subspace of codimension 1 and assume that $E$ contains Chebyshev systems of arbitrary high dimensions. Then $R(E)$ is dense in $C([0,1])$.

Proof. Let $E_{n}$ be a Chebyshev System of dimension $n$. It is well-known that for every given $f \in C([0,1])$ there exists a unique best approximation $g \in E_{n}$ to $f$. Moreover, there are $n+1$ points $x_{0}<x_{1}<\cdots<x_{n} \in$ $[0,1]$ such that either $\|f-g\|=(-1)^{i}\left(f\left(x_{i}\right)-g\left(x_{i}\right)\right)$ or $\|f-g\|=$ $(-1)^{i+1}\left(f\left(x_{i}\right)-g\left(x_{i}\right)\right)$ holds for all $0 \leq i \leq n$. Now assume that $E=\operatorname{ker} \mu$ and assume that $R(E)$ is not dense in $C(K)$. Then supp $\mu_{+} \cap \operatorname{supp} \mu=\varnothing$ (see [3]). Pick a continuous function $f$ such that $\|f\|=1$ and such that $f(k)=1$ for $k \in \operatorname{supp} \mu_{+}$and $f(k)=-1$ for $k \in \operatorname{supp} \mu_{-}$. We then have $f \mu=|\mu|$. Let $E_{n} \subset E$ be a Chebyshev subspace of dimension $n$ and let $g_{n} \in E_{n}$ be the best approximation to $f$. Since $\mu\left(g_{n}\right)=0$, it follows that

$$
\begin{aligned}
\left\|f-g_{n}\right\|\|\mu\| & \geq\left|\mu\left(f-g_{n}\right)\right|=|\mu(f)|=|(f \mu)(1)|=|\mu|(1) \\
& =\|\mu\|=\|\mu\|\|f\|
\end{aligned}
$$

and hence $\|f\|=\|f-0\| \leq\left\|f-g_{n}\right\|$. Since the best approximation of $f$ is unique, it follows that $0=g_{n}$. Hence we can find $n+1$ points $x_{0}, \ldots, n$ such that $f\left(x_{i}\right)=(-1)^{i}\|f\|=(-1)^{i}$. This last statement is true for arbitrary large $n$, contradicting the continuity of $f$. 
A well-known example of a space $E$ containing Chebyshev subspaces of arbitrary high dimension without being dense can be obtained as follows: Let

$$
E=\operatorname{span}\left\{\frac{1}{t-\lambda_{J}}: j=0,1,2, \ldots\right\} \subset C([0,1])
$$

where $\Lambda=\left\{\lambda_{j}: j=0,1,2, \ldots\right\} \subset(-\infty, 0) \cup(1, \infty)$ is an infinite set of real numbers such that $\Lambda \cap[0,1] \neq \varnothing$. It turns out that for every measure $\mu \perp E$ either 0 or 1 always belong to supp $\mu_{+} \cap \operatorname{supp} \mu_{-}$, depending on whether $0 \in \bar{\Lambda}$ or $1 \in \bar{\Lambda}$. Hence $R(E)$ is dense in $C([0,1])$. However, there is a simpler proof for this fact: It is enough to show that every polynomial $P_{n}(t)=\sum_{l=0}^{n} a_{j} t^{j}$ belongs to $R(E)$. By partial fractions

$$
g(t)=\frac{P_{n}(t)}{\prod_{j=1}^{n+1}\left(t-\lambda_{j}\right)} \in E
$$

and

$$
h(t)=\frac{1}{\prod_{J=1}^{n+1}\left(t-\lambda_{j}\right)} \in E .
$$

Hence $g(t) / h(t)=P_{n}(t) \in R(E)$.

\section{REFERENCES}

[1] J. Bak and D. J. Newman, Rational combinations of $x^{\lambda_{k}}, \lambda_{k} \geq 0$ are always dense in $C([0,1])$, J. Approximation Theory, 32, No. 2 (1978), 155-157.

[2] R. P. Feinerman, and D. J. Newman, Polynomial Approximation, Williams \& Wilkins Co., Baltimore (1974).

[3] G. Gierz, and B. Shekhtman, On rational approximation from a hyperplane, Proc. Amer. Math. Soc. to appear.

[4] D. J. Newman, Approximation with Rational Functions, CBMS Regional Conference Series in Mathematics, No. 28 (1979).

[5] G. Somorjai, A Müntz-type problem for rational approximation, Acta Math. Acad. Hungar., 27 (1976), 197-199.

[6] R. S. Varga, Topics in Polynomial and Rational Interpolation and Approximation, Séminaire de Mathématiques Supérieures, Les Presses de 1'Université de Montréal (1982).

Received September 6, 1985 and in revised form January 10, 1986.

DePartMent of Mathematics

UNIVERSITY OF CALIFORNIA

RIVERSIDE, CA 92521 


\section{PACIFIC JOURNAL OF MATHEMATICS EDITORS}

\author{
V. S. VARADARAJAN \\ (Managing Editor) \\ University of California \\ Los Angeles, CA 90024 \\ HERBERT ClEMENS \\ University of Utah \\ Salt Lake City, UT 84112 \\ R. FINN \\ Stanford University \\ Stanford, CA 94305
}

\author{
HERMANN FLASCHKA \\ University of Arizona \\ Tucson, AZ 85721 \\ RAMESH A. GANGOLLI \\ University of Washington \\ Seattle, WA 98195 \\ VAUghan F. R. JONES \\ University of California \\ Berkeley, CA 94720 \\ ROBION KIRBY \\ University of California \\ Berkeley, CA 94720
}

C. C. MOORE

University of California Berkeley, CA 94720

H. SAMELSON

Stanford University Stanford, CA 94305

HAROLD STARK

University of California, San Diego La Jolla, CA 92093

\section{ASSOCIATE EDITORS}
R. ARENS
E. F. BECKENBACH
B. H. NEUMANN
F. WOLF
K. YOSHIDA (1906-1982)

\section{SUPPORTING INSTITUTIONS}

UNIVERSITY OF ARIZONA

UNIVERSITY OF BRITISH COLUMBIA

CALIFORNIA INSTITUTE OF TECHNOLOGY

UNIVERSITY OF CALIFORNIA

MONTANA STATE UNIVERSITY

UNIVERSITY OF NEVADA, RENO

NEW MEXICO STATE UNIVERSITY

OREGON STATE UNIVERSITY
UNIVERSITY OF OREGON UNIVERSITY OF SOUTHERN CALIFORNIA

STANFORD UNIVERSITY

UNIVERSITY OF HAWAII

UNIVERSITY OF TOKYO

UNIVERSITY OF UTAH

WASHINGTON STATE UNIVERSITY

UNIVERSITY OF WASHINGTON 


\section{Pacific Journal of Mathematics}

Vol. 125, No. 1 September, 1986

Gilles Christol, Fonctions et éléments algébriques $\ldots \ldots \ldots \ldots \ldots \ldots \ldots \ldots$

Jo-Ann Deborah Cohen, Extensions of valuation and absolute valued

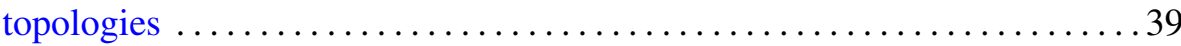

Miriam Cohen, Smash products, inner actions and quotient rings . . . . . . 45

Mikio Furushima, On the singular $K-3$ surfaces with hypersurface

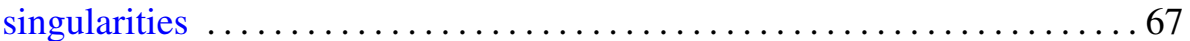

Gerhard Gierz and Boris Shekhtman, A duality principle for rational

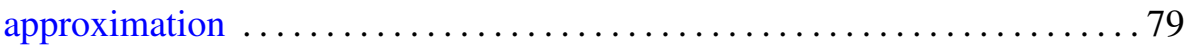

Anthony Wood Hager, A description of HSP-like classes, and

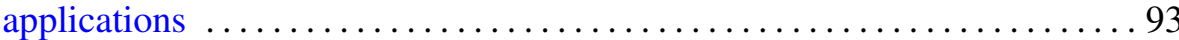

George Alan Jennings, Lines having high contact with a projective

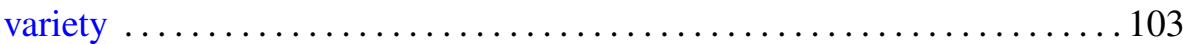

John Lott, Eigenvalue bounds for the Dirac operator . . . . . . . . . . . 117

Denis Laurent Luminet, A functional calculus for Banach PI-algebras . . . . 127

Shizuo Miyajima and Noboru Okazawa, Generators of positive

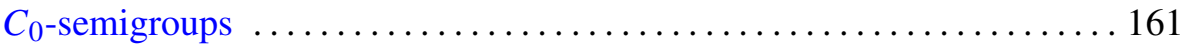

Takemi Mizokami, On functions and stratifiable $\mu$-spaces $\ldots \ldots \ldots \ldots \ldots 177$

Jeff Parker, 4-dimensional $G$-manifolds with 3-dimensional orbits . . . . . 187

Elias Saab and Paulette Saab, On Peł czyński's properties (V) and (V*) . . 205

Elmar Schrohe, The symbols of an algebra of pseudodifferential operators

Aart van Harten and Els Vader-Burger, Approximate Green functions as a tool to prove correctness of a formal approximation in a model of competing and diffusing species

Stephen Watson, Using prediction principles to construct ordered

continua 